\title{
High-Field Half-Cycle Terahertz Radiation from Relativistic Laser Interaction with Thin Solid Targets
}

\author{
W. J. Ding ${ }^{1}$, Z. M. Sheng ${ }^{2,3}$, W. S. Koh ${ }^{1}$ \\ ${ }^{1} A *$ STAR Institute of High Performance Computing, Singapore 138632 \\ ${ }^{2}$ Key Laboratory for Laser Plasmas (MoE) and Department of Physics and Astronomy, \\ Shanghai Jiao Tong University, Shanghai 200240, China \\ ${ }^{3} S U P A$, Department of Physics, University of Strathclyde, Glasgow G4 0NG, UK
}

\begin{abstract}
It is found that half-cycle terahertz $(\mathrm{THz})$ pulses with the peak field over $100 \mathrm{MV} / \mathrm{cm}$ can be produced in ultrashort intense laser interactions with a thin solid target. These THz pulses are shown to emit from both the front and rear sides of the solid target and are attributed to the coherent transition radiation (CTR) by laserproduced ultrashort fast electron bunches. After the primary THz pulses, subsequent secondary half-cycle pulses are generated while some refluxing electrons cross the vacuum-target interfaces. Since such strong THz radiation is well synchronized with the driving lasers, it is particularly suitable for applications in various pump-probe experiments.
\end{abstract}

PACS number: 52.35.Mw, 52.25.Os, 52.59.Ye, 52.38.-r 
In the last decade, there has been significant interest in producing strong terahertz (THz) radiation with field strength as high as over $1 \mathrm{MV} / \mathrm{cm}$, either by use of short electron bunches or by use of ultrashort laser pulses [1,2]. Typically one can produce few-cycle strong THz sources, which are particularly interesting for nonlinear and non-equilibrium studies, including $\mathrm{THz}$ nonlinear optics, $\mathrm{THz}$ pump experiments on coherent control of structure dynamics of various matters, control of chemical reactions, high magnetic field effects, etc. $[3,4]$. For $\mathrm{THz}$ radiation produced by femtosecond laser-gas interaction, a well-known mechanism is the ionization current, which can be effectively produced by two-color lasers or by few cycle lasers $[5,6]$. High field THz emission can also be obtained from laser wakefield excitation in underdense plasma via mode conversion [7,8]. Via coherent transition radiation (CTR), high field THz radiation with ultrashort electron bunches from Linac has been reported $[9,10,11]$. In principle, the ultrashort intense laser pulses can also generate ultrashort electron bunches, which can be used to produce intense $\mathrm{THz}$ emission through CTR. It has been reported that the ultrashort electron bunches accelerated in the laser wakefield in underdense plasma can emit strong THz emission at the plasmavacuum boundary via the CTR mechanism [12]. It was also observed in experiments that the interaction of ultrashort intense laser pulses with solid targets can produce strong $\mathrm{THz}$ radiation $[13,14]$.

In this Letter, we show via self-consistent numerical simulation that half-cycle THz pulses with extremely high amplitudes can be produced by ultrashort intense lasers irradiating on a thin solid target. Both forward and backward radiations are observed from the front and rear sides of the target. We attribute these THz pulses to CTR by ultrashort hot electron bunches produced in laser-solid interactions crossing surfaces of the target. 


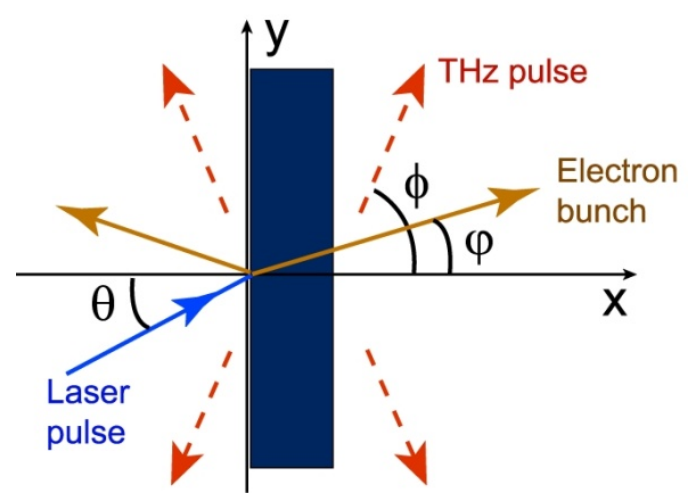

Fig. 1. (Color online) Schematic of THz radiation produced by laser-interaction with a thin foil.

The schematic of the laser-solid interaction and resulting $\mathrm{THz}$ emission is shown in Fig. 1, where an ultrashort intense laser is incident onto the solid target with the angle $\theta$. Under the laser irradiation, fast electrons are produced at a dominant angle $\varphi$ via different mechanisms such as laser ponderomotive force acceleration [15], vacuum heating [16], resonance absorption [17], etc. Generally, there are two major groups of fast electrons, with one emitting backward into the vacuum through the preformed underdense plasma and another emitting forward into the target. If a thin foil target is adopted, the fast electrons can be found both in the front and rear sides. Electromagnetic radiation can be produced as a bunch of fast electrons passes through the target-vacuum interface via transition radiation (TR) as shown theoretically by Zheng et al. [18]. This radiation appears to be coherent for the frequency $\omega<2 \pi v / L_{e}$, where $v$ is the electron bunch velocity and $L_{e}$ is the bunch length.

When a single test electron crossing the interface of solid plasma-vacuum, the TR spectrum (in the plane of incidence) is $\frac{d^{2} \mathcal{E}}{d \omega d \Omega}=\frac{e^{2}}{\pi^{2} c} \frac{[\beta \cos \varphi(\sin \phi-\beta \sin \varphi)]^{2}}{\left[(1-\beta \sin \phi \sin \varphi)^{2}-(\beta \cos \phi \cos \varphi)^{2}\right]^{2}}$, where $\varphi$ is the incident of angle of the test electron, $\phi$ the emission angle of TR, and $\beta$ is the velocity of the electron normalized by the speed of light in vacuum $c$ [18]. TR vanishes in the angle $\phi_{0}=\arcsin (\beta \sin \varphi)$. The radiation intensity has two lobes with two maxima around the angle $\phi_{0}$. In the nonrelativistic limit, the emission peaks 
along the surface of the solid target at $\phi= \pm 90^{\circ}$. Note that the TR spectrum from a single electron is independent of the frequency, whereas for an electron bunch, the emission spectrum from CTR is closely related to its specific energy and angular distribution [18]. In the case of relativistic laser-solid interaction, hot electrons usually have a thermal energy distribution featuring two or more temperatures. The angular distribution of hot electrons is also complex [19]. Thus the spectrum is not easily described analytically. On the other hand, since the electron bunches produced from relativistic laser solid interaction usually have a bunch length in the order of the laser pulse duration, it is possible to observe coherent emission from TR in the THz range using sub-picosecond lasers. In the following, we examine this by particle-incell (PIC) simulation.

We consider a laser pulse with the temporal and spatial profile given by $a=$ $e A / m_{e} c^{2}=a_{0} \sin ^{2}\left(\frac{\pi t}{T}\right) \exp \left(-\frac{y^{2}}{w_{0}^{2}}\right)$, where the peak amplitude of the normalized vector potential $a_{0}$ is related to the laser intensity $I \lambda_{0}^{2}=a_{0}^{2} \times 1.37 \times 10^{18} \mathrm{~W} /$ $\mathrm{cm}^{2} \mu \mathrm{m}^{2}, \lambda_{0}$ is the laser wavelength in vacuum and set as $800 \mathrm{~nm}, w_{0}$ is relative to FWHM beam radius via $w_{F W H M}=2 \sqrt{\ln 2} w_{0}$. We use a thin target composed of a high density platform at $10 n_{c}$ (with $n_{c}$ the critical density $n_{c}=m \omega_{0}^{2} / 4 \pi e^{2}$ ) and the preplasma exponentially increases along the $x$ axis from $0.1 n_{c}$. The scale of the preplasma is crucial for the generation of hot electrons as too long or short preplasma is unfavorable for the THz emission generation [13]. the optimal scale length of the preplasma is $L=0.5 \lambda_{0}$. The target with a thickness $D=11 \lambda_{0}$ starts from $x=0$. The laser pulse is p-polarized with the optimal incident angle $\theta=20^{\circ}$ depending on the scale length $L$. The spatial step $D x=D y=1 / 50 \lambda_{0}$ and the time step $D t=1 / 100 \lambda_{0} / c$ in the simulations. 

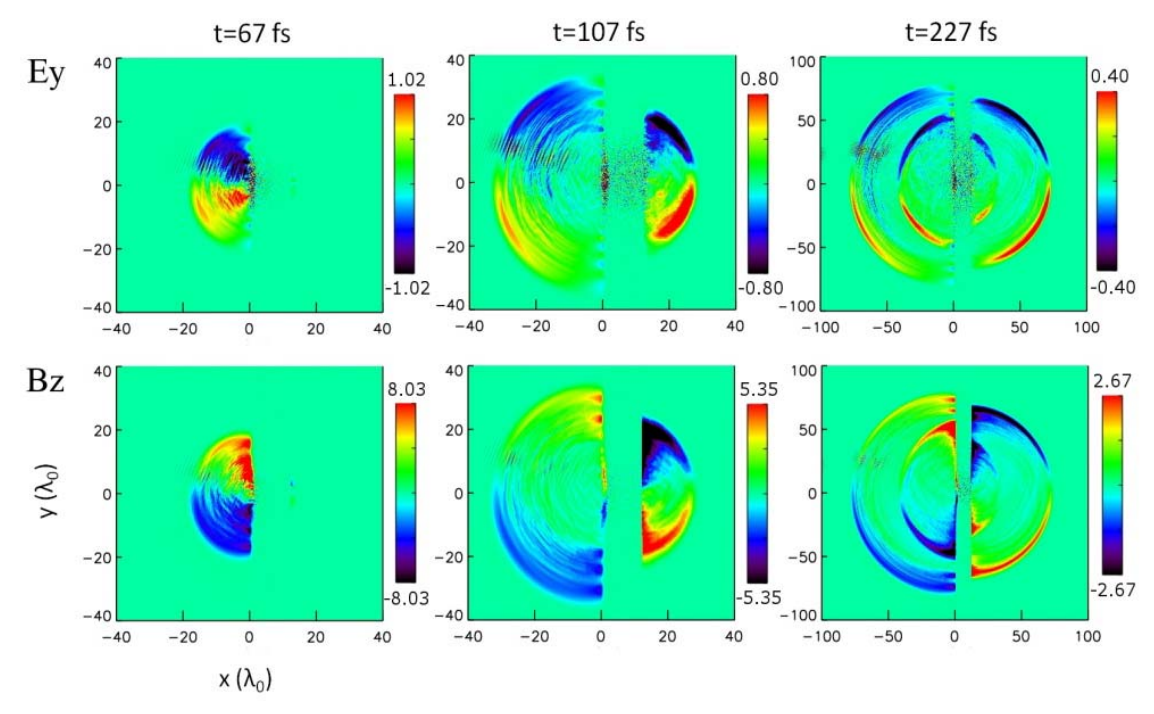

Fig. 2. (Color online) Snapshots of electric and magnetic fields time-averaged in a laser cycle $\tau_{0}$. The incident laser has the peak amplitude $a_{0}=0.6\left(I=7.7 \times 10^{17} \mathrm{~W} / \mathrm{cm}^{2}\right)$, duration $T=80 \mathrm{fs}, w_{0}=5 \lambda_{0}=4.0 \mu \mathrm{m}$. Here the field $\mathrm{E}_{\mathrm{y}}$ is in unit of $\mathrm{GV} / \mathrm{cm}$ and $\mathrm{B}_{\mathrm{z}}$ in $\mathrm{MG}$.

Figure 2 shows snapshots of the slowly-varying transverse electric and magnetic fields. At the beginning, the low frequency emission is found only at the laserirradiated front side of the target. At $t=107 \mathrm{fs}$, it is also found at the rear side. When $t=227 \mathrm{fs}$, the radiation can be seen in the whole $2 \pi$ space, which emits from two point sources around the laser irradiation spot at the front and rear sides, respectively. Note that the polarity of transverse electric field and magnetic field are the same on the front side, but reversed on the back side of the target. These field distributions indicate that they are associated with the electrons emitting to the left at the front surface and to the right at the rear surface.

It is observed that the strongest emission is along the target surfaces. The peak field amplitude is easily over $100 \mathrm{MV} / \mathrm{cm}$ in a distance $100 \lambda_{0}$ away from the target surface. Since the source size can be considered as a point, the field amplitude is estimated to decay in terms of $1 / R$ with $R$ the distance from the laser irradiation position. The pulse energy is estimated to be over $0.1 \mathrm{~mJ}$ in the present simulation. There is only a small region, near the angle of $10^{\circ}$ to the normal direction, where the radiation vanishes. Similar spatial distribution of $\mathrm{THz}$ radiation is recently measured 
from the target back [14], and it is reported that a tiny hollow zone locates at the center and the peak at the far end from the center. This kind of angular distribution is unsurprisingly similar to that of TR from a weakly relativistic electron [18]. As shown in previous theory and simulation [19,20], the fast electrons have thermal energy distribution and are emitted between the target normal and the specular reflection direction (at the front) or the incident laser direction (at the rear side). Combining the angular distribution of hot electrons and TR, one expects to observe the field distribution shown in Fig. 2. Before $t=227 \mathrm{fs}$, second pulses occur in both forward and backward radiation. This suggests that multiple electron bunches are produced at the front and rear sides.
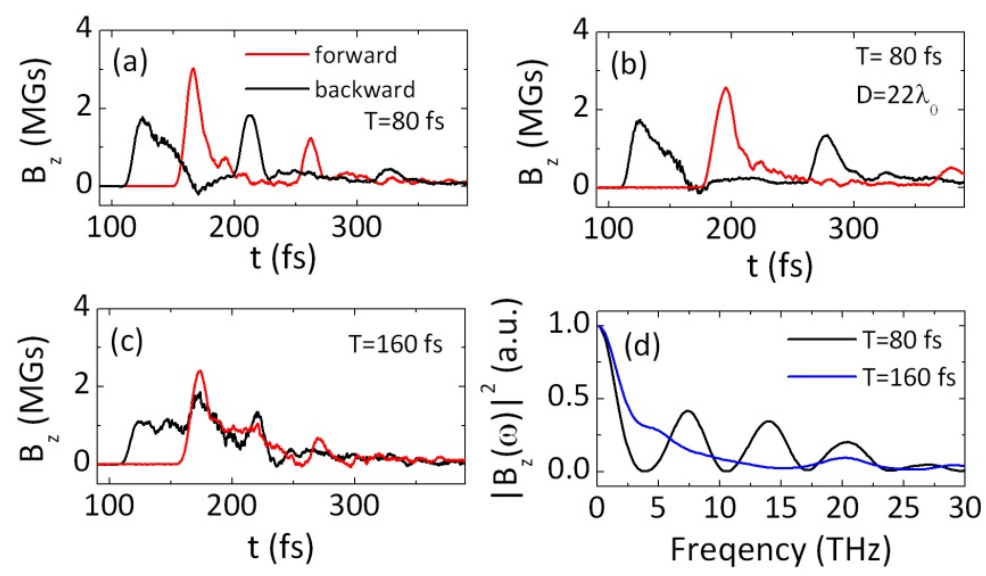

Fig.3. (Color online) Temporal profiles of radiations (Bz averaged in a laser cycle) detected at two typical points in front and back of the target at $40 \lambda_{0}$ away from the emission spots for three cases with $T=80$ or $160 \mathrm{fs}$, and $D=12 \lambda_{0}$ or $22 \lambda_{0}$ [only in (b)]. (d) Spectra of backward radiations in (a) and (c). Other parameters are the same as Fig.2.

To see the structures of the low frequency emission, the temporal profiles emitted along the direction $\phi=60^{\circ}$ and the spectra are illustrated in Fig. 3. Shortly after the laser pulse irradiation, half-cycle low frequency radiations are generated. The duration 
of the first or primary half-cycle pulse is close to the incident laser duration $T$. An interesting phenomenon observed is the presence of secondary half-cycle pulses after the primary pulses. The secondary pulses are emitted after the laser pulse reflected from the target, i.e. after the laser interaction, and they appear to have shorter duration than the primary pulses. Another interesting aspect is that the time separation between the primary and secondary half-cycle pulses is slightly larger than $2 D / c$, or twice the time separation of pulses emitted at the front and rear side. Note that $2 D / c$ is approximately the time required for the fast electrons to move from the front to the rear and then return to the front. This suggests that the secondary half-cycle pulses are produced from the refluxing hot electrons. Quasi-static charge separation field produced near the target surfaces tends to pull some of the less energetic electrons back into the target, while the very energetic ones leave the target directly. Note that electron bunch recirculation has been observed experimentally via CTR in intense laser solid interactions [21].

When the target thickness is increased by $\Delta D=10 \lambda_{0}$ [see Fig. 3(b)], the time delay for the secondary pulses is also increased by about $2 \Delta \mathrm{D} / \mathrm{c}$. As the laser intensity increases, the increase in both the energy and number of hot electrons leads to the enhancement of the $\mathrm{THz}$ radiation correspondingly. When the incident laser pulse duration is increased [see Fig. 3(c)], longer half-cycle pulses are produced, thus the primary half-cycle pulses can overlap with the secondary pulses. Consequently the radiation appears to have a sharp front and then a slower decaying tail for forward radiations. The radiation spectra are also shown in Fig. 3(d) with different laser durations. Besides the $\mathrm{DC}$ components near the frequency $f=0$, which are due to the half-cycle shape of the pulses, the spectra peaks at $f=7.5$ and $3.7 \mathrm{THz}$ for $T=80$ and $160 \mathrm{fs}$, respectively. These are in agreement with the length of the primary THz pulses shown in Figs. 3(a) and 3(c), which are 66.7 and 133 fs, respectively. There are also higher frequency components in the spectrum due to the shorter secondary pulses. Taking Fig. 3(c) for an example, the incident laser pulse is $160 \mathrm{fs}$ long (80fs of FWHM) with intensity $7.7 \times 10^{17} \mathrm{~W} / \mathrm{cm}^{2}$. The obtained $\mathrm{THz}$ radiations are about $133 \mathrm{fs}$, corresponding to a central frequency $3.7 \mathrm{THz}$. The emission reaches the intensity 
$\sim 10^{14} \mathrm{~W} / \mathrm{cm}^{2}$ in the near field region, which is much higher than other THz sources reported so far.
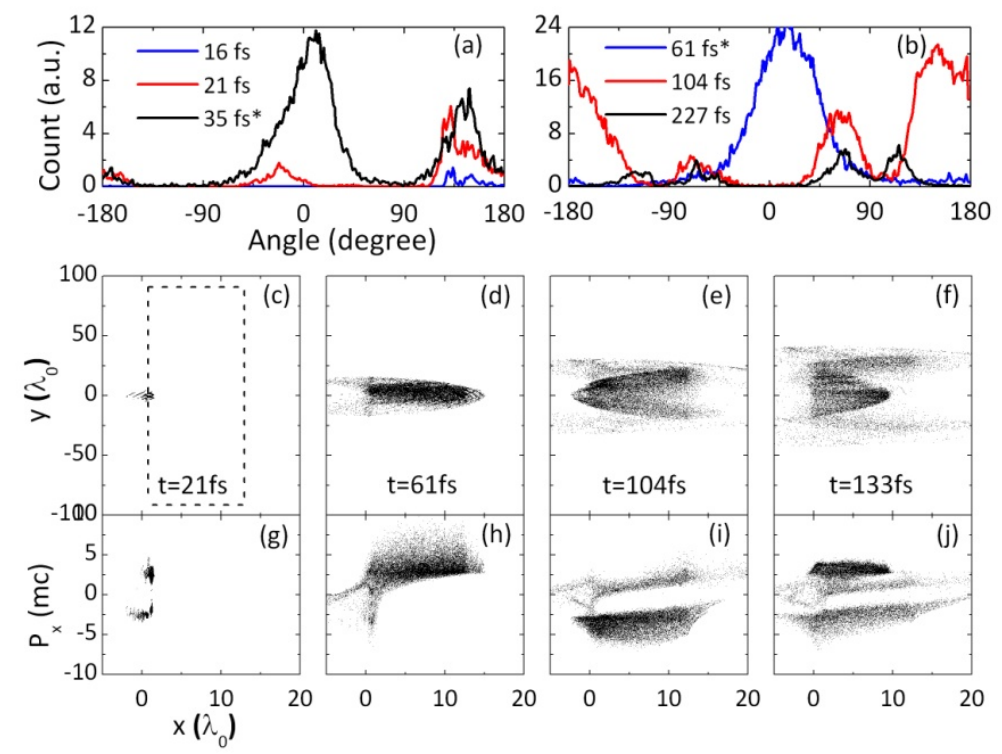

Fig. 4. (Color online) Angular (a-b), spatial (c-f) and phase (g-j) distributions of fast electrons $\left(E_{k}>0.8 \mathrm{MeV}\right)$ within and near the target at different times. Angle defined as $\tan ^{-1}\left(p_{y} / p_{x}\right)$. Count of electrons at $t=35$ and $61 \mathrm{fs}$ multiplied by 0.5 for better illustrations with other times. The target is marked by dash rectangular in (c). Except for $a_{0}=1.5$, other parameters are the same as Fig.2.

As mentioned above, the fast electron generation is responsible for the CTR in our case. In the following, we discuss the evolution of fast electrons distributions in order to understand the CTR further. In the ultrashort laser interaction with a solid target, a small preplasma with a scalelength $L<\lambda_{0}$ can be produced if the laser contrast ratio is controlled properly [22]. In this case, hot electrons are mainly generated by ponderomotive force, vacuum heating, and resonance absorption. Tracing of individual electrons shows some electrons are accelerated and decelerated many times in and out of the target in the presence of the laser field. The collective behaviors of hot electrons are illustrated in Figs. 4(a) and 4(b). At the very beginning $t=16 \mathrm{fs}$, backward electrons are produced at the front surface of the target. Shortly later $t=21 \mathrm{fs}$ electrons gain energy forward via ponderomotive force as laser intensity grows. Then 
forward-moving electrons dominate inside the target, as shown for $t=35 \mathrm{fs}$ and $61 \mathrm{fs}$. At the rear half duration of the laser, backward-moving electrons increase. At $t=104 \mathrm{fs}$ when the laser leaves the target, there are both backward and forward electrons near and inside the target. Less hot electrons are pulled back by sheath fields near the target surfaces, some of which are confined by the quasi-static fields in a long time oscillating in and out of the target while drifting towards the ends of the target, as shown for $t=227 \mathrm{fs}$. Spatial and phase plots of hot electrons also show the evolution of electron bunches. In the beginning, both forward and backward hot electrons are found at the front surface of the target [Fig.4(c) and 4(g)]. Subsequently, forward ones cross the rear side [Fig.4(d)and 4(h)] producing the first forward THz pulse, and most of them are pulled back by the static field near the rear surface and traverse the front surface [Fig.4(e) and 4(j)] producing the second backward THz pulse. Then, the majority of those electrons are dragged back by the static field near the front surface [Fig.4 (f) and 4(j)] generating the second forward THz pulse.

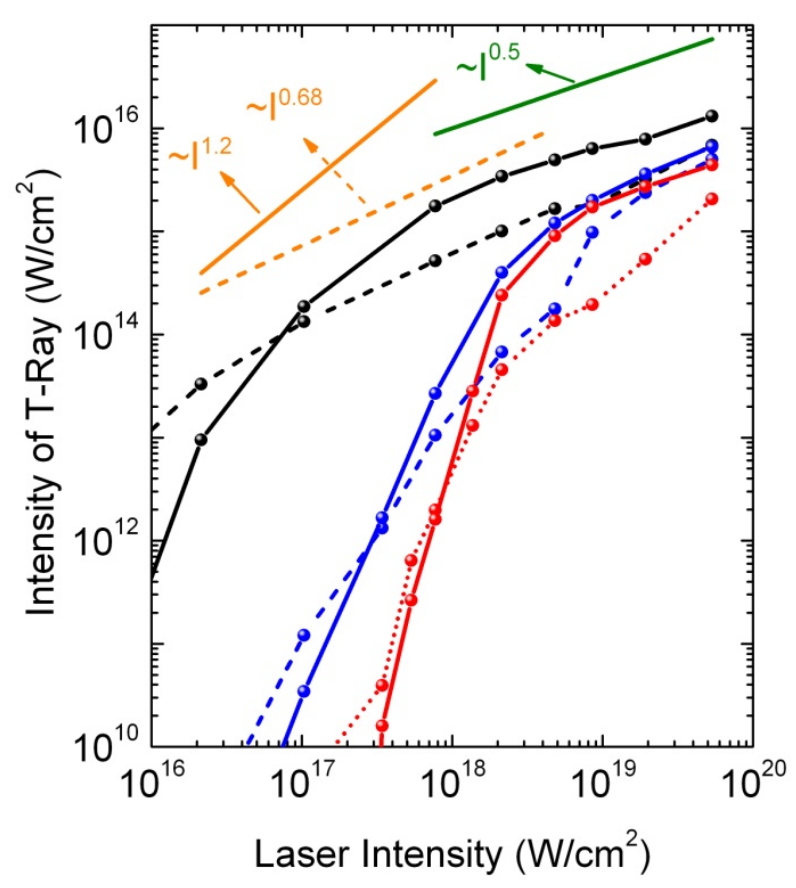

Fig. 5. (Color online) Intensity of THz radiations (only initial pulses) near the target surface under different laser incidence conditions: oblique incidence $\left(\theta=20^{\circ}\right)$ of $\mathrm{p}$ polarized laser (black lines), normal incidence of p-polarized laser (blue lines), and 
oblique incidence of s-polarized laser (red lines). Other parameters are fixed to be the same as Fig. 2. Solid and dashed lines are for forward and backward radiations, respectively. The straight green and orange lines are shown to guide the eyes for different scalings.

We have compared the radiation intensity with three different irradiation conditions, either normal or oblique incidence of p- or s-polarized lasers [see Fig. 5]. The intensity of $\mathrm{THz}$ radiations increases monotonously with laser intensity. The most intense radiation is produced by oblique incidence of p-polarized lasers, simply because hot electrons are most efficiently produced when the vacuum heating, resonance absorption, and ponderomotive force acceleration all play roles in this case. For weak lasers when the ponderomotive force is small, resonance absorption becomes dominant and the emitted radiation is more than 3 orders higher than other two cases. As the laser intensity increases, ponderomotive force dominates in the fast electron generation, the difference between the three cases decreases. When the laser intensity reaches the relativistic regime $\left(>2.1 \times 10^{18} \mathrm{~W} / \mathrm{cm}^{2}\right.$ for $\left.\lambda_{0}=800 \mathrm{~nm}\right)$, the $\mathrm{THz}$ radiations found at different incident conditions are basically in the same order with the scaling $I_{T H z} \sim I^{1 / 2}$, indicating that the ponderomotive force effect becomes dominant for hot electron generation for all cases. This is in agreement with the scaling on fast electron number and temperature with the laser intensity $[19,23,24]$. One may also notice that the forward radiations are usually more intense than backward radiations because there are more energetic forward moving electrons than backward ones and there is preplasma at the front with low density, which tends to reduce the CTR.

Even though the THz pulses shown above are half-cycle, it is worthwhile to point out that they can also be single cycle under different conditions. In the case when there is no or weak resonance absorption or vacuum heating such as under normal incidence or large angles of incidence or using s-polarized lasers, the primary pulse of the backward radiation is in single-cycle with a weak half-cycle ahead. This is because the ponderomotive force will first produce forward acceleration of hot 
electrons at the front surface, which generate a half-cycle CTR. Later at the trailing edge of the laser pulse the second half-cycle of the emission generated by backward electrons appears with a different sign. In the case when resonance absorption or vacuum heating happens, backward electrons are immediately produced. Then the emission with a different sign by forward electrons becomes not obvious and only a half-cycle pulse is seen. In some reported experiments, single-cycle shaped pulses were observed for backward THz radiation with a large incident angle [13]. Our simulation with such a large incident angle shows that less fast electrons are produced as compared to that for the optimal incident angle shown above. The resulting electric field of the radiation is about half and $20 \%$ of those obtained at the optimal incidence angle for backward and forward radiation, respectively. The single-cycle emission can be explained as due to the presence of subsequent forward and backward currents crossing the front surface.

In summary, we have demonstrated via PIC simulations that high field half-cycle THz pulses can be produced both in forward and backward directions by ultrashort intense laser interaction with a thin solid target with moderate laser intensity such as $10^{17} \mathrm{~W} / \mathrm{cm}^{2}$. The emission is attributed to CTR when ultrashort electron bunches produced in the laser interaction transmit through the target-vacuum interfaces. Furthermore, the refluxing electrons pulled back into the target by the quasi-static fields near the target surfaces can produce additional half-cycle pulses subsequently. Our proposed scheme is compact, offering a route to produce ultra-strong $\mathrm{THz}$ sources for applications such as pump-probe high field $\mathrm{THz}$ irradiation experiments. Moreover, it provides a unique way to understand the transport of a large flux of electron beam transport in a dense plasma target, which is potentially important for high energy density physics driven by ultrashort high intensity lasers.

WJD thanks Y.T. Li, C. Li, M.L. Zhou and W.M. Wang from IoP-CAS for enlightening discussions on their experiments. ZMS acknowledges the support by the National Basic Research Program of China (Grant No. 2013CBA01504) and NSFC 
(Grant No. 11121504, 11129503, and 11075105). This work was supported by the A*STAR Computational Resource Centre through the use of its high performance computing facilities.

\section{References}

1. G. P. Williams, Rep. Prog. Phys. 69, 301 (2006).

2. K. Reimann, Rep. Prog. Phys. 70, 1597 (2007); H. G. Roskos, M. D. Thomson, M. Kreß and T. Löffler Laser \& Photon. Rev. 1, 349 (2007).

3. M. S. Sherwin, C. A. Schmuttenmaer, and P. H. Bucksbaum, Ed. Opportunities in THz Science, Report of a DOE-NSF-NIH Workshop held February 12 - 14, 2004, Arlington, VA.

4. Y. Shen, T. Watanabe, D. A. Arena, C.-C. Kao, J. B. Murphy, T. Y. Tsang, X. J. Wang, and G. L. Carr, Phys. Rev. Lett. 99, 043901 (2007); M. C. Hoffmann and J. A. Fülöp, J. Phys. D 44, 083001(2011).

5. K. Y. Kim, J. H. Glownia, A. J. Taylor, and G. Rodriguez, Opt. Express 154577 (2007).

6. H. C. Wu, J. Meyer-ter-Vehn, and Z. M. Sheng, New J. Phys. 10, 043001(2008); M. Chen, A. Pukhov, X.-Y. Peng, and O. Willi, Phys. Rev. E 78, 046406 (2008).

7. H. Hamster, A. Sullivan, S. Gordon, W. White, and R. W. Falcone, Phys. Rev. Lett. 71, 2725 (1993).

8. Z. M. Sheng K. Mima, J. Zhang, and H. Sanuki, Phys. Rev. Lett. 94, 095003 (2005); H. C. Wu, Z. M. Sheng, Q. L. Dong, H. Xu, and J. Zhang, Phys. Rev. E 75,016407 (2007).

9. N. S. Ginzburg, I. V. Zotova, A. S. Sergeev, V. Yu. Zaslavsky, and I. V. Zheleznov, Phys. Rev. Lett. 108, 105101 (2012).

10. Z. Wu , A. S. Fisher, J. Goodfellow, M. Fuchs, D. Daranciang, M. Hogan, H. Loos, and A. Lindenberg, Rev. Sci. Instrum. 84, 022701 (2013). 
11. D. Daranciang J. Goodfellow, M. Fuchs, H. Wen, S. Ghimire, D. A. Reis, H. Loos, A. S. Fisher, and A. M. Lindenberg, Appl. Phys. Lett. 99, 141117 (2011).

12. W. P. Leemans, C.G. R. Geddes, J. Faure, Cs. Tóth, J. van Tilborg, C. B. Schroeder, E. Esarey, G. Fubiani, D. Auerbach, B. Marcelis, et al., Phys. Rev. Lett. 91, 074802 (2003); J. van Tilborg C. B. Schroeder, C. V. Filip, Cs. Tóth, C. G. R. Geddes , G. Fubiani , E. Esarey, and W. P. Leemans, Phys. Plasmas 13, 056704 (2006) .

13. Y. T. Li, C. Li , M. L. Zhou, W. M. Wang, F. Du, W. J. Ding, X. X. Lin, F. Liu, Z. M. Sheng, X. Y. Peng et al., Appl. Phys. Lett. 100, 254101 (2012); C. Li, M. L. Zhou, W. J. Ding, F. Du, F. Liu, Y. T. Li, W. M. Wang, Z. M. Sheng, J. L. Ma, L. M. Chen et al., Phys. Rev. E 84, 036405 (2011).

14. A. Gopal, T. May, S. Herzer, A. Reinhard, S. Minardi, M. Schubert, U. Dillner, B. Pradarutti, J. Polz, T. Gaumnitz et al., New J. Phys. 14, 083012 (2012).

15. C. E. Max and J. Arons, Phys. Rev. Lett. 33, 209 (1974).

16. F. Brunel, Phys. Rev. Lett. 59, 52 (1987).

17. K. G. Estabrook, E. J. Valeo, and W. L. Kruer, Phys. Fluids 18, 1151(1975); W. J. Ding, Z. M. Sheng, J. Zhang, and M. Y. Yu, Phys. Plasma 16, 042315 (2009).

18. J. Zheng K. A. Tanaka, T. Miyakoshi, Y. Kitagawa, R. Kodama, T. Kurahashi, and T. Yamanaka, Phys. Plasmas 10, 2994 (2003).

19. Z. M. Sheng, Y. Sentoku, K. Mima, J. Zhang, W. Yu, and J. Meyer-ter-Vehn, Phys. Rev. Lett. 85, 5340 (2000).

20. M. Chen Z. M. Sheng, and J. Zhang, Phys. Plasmas 13, 014504 (2006).

21. S. D. Baton, J. J. Santos, F. Amiranoff, H. Popescu, L. Gremillet, M. Koenig, E. Martinolli, and O. Guilbaud, Phys. Rev. Lett. 91, 105001 (2003).

22. Y. Ping, R. Shepherd, B. F. Lasinski, M. Tabak, H. Chen, H. K. Chung, K. B. Fournier, S. B. Hansen, A. Kemp, D. A. Liedahl et al., Phys. Rev. Lett. 100, 085004 (2008).

23. S. C. Wilks, W. L. Kruer, M. Tabak, and A. B. Langdon, Phys. Rev. Lett. 69, 1383 (1992). 
24. Y. Q. Cui, W. M. Wang, Z. M. Sheng, Y. T. Li, and J. Zhang, Plasma Phys. Control. Fusion 55, 085008 (2013). 